\title{
Extreme scale-dependent tensile properties of epoxy fibers
}

\author{
X. M. Sui ${ }^{1}$, M. Tiwari ${ }^{1,2}$, I. Greenfeld ${ }^{1}$, R. L. Khalfin ${ }^{3}$, H. Meeuw ${ }^{4}$, B. Fiedler ${ }^{4}$, H. D. Wagner $^{1 *}$ \\ ${ }^{1}$ Department of Materials and Interfaces, Weizmann Institute of Science, 7610001 Rehovot, Israel \\ ${ }^{2}$ Department of Mechanical Engineering, Indian Institute of Technology Ropar, 140001 Rupnagar, India \\ ${ }^{3}$ Department of Chemical Engineering, Technion, Israel Institute of Technology, Haifa 3200003, Israel \\ ${ }^{4}$ Institute of Polymers and Composites, Hamburg University of Technology, D-21073 Hamburg, Germany
}

Received 4 March 2019; accepted in revised form 18 June 2019

\begin{abstract}
Epoxy fibers with different diameters were prepared by hot drawing and their mechanical properties were measured under tension. The stiffness, strength, ultimate strain, and toughness revealed substantial scale-dependent effects as they all significantly increased with a decrease in size. Compared to bulk epoxy, an intrinsically brittle material, thin epoxy fibers displayed a highly ductile behavior under tension. A drop in stress observed immediately beyond the yield point was followed by the development of a stable necking region propagating through the entire fiber length, then by strain-hardening up to final rupture. Necked fiber segments tested in tension were found to have even higher strength and modulus compared to the initial as-prepared fibers. Possible reasons for the highly ductile mechanical behavior and the size effects of epoxy fibers are discussed. Size effects for the strength of epoxy can be elucidated in principle either by means of a classical fracture mechanics argument (strength $\sim 1 / d^{1 / 2}$ ), or via a stochastic model argument (strength $\sim 1 / d^{1 / \beta}$, where $\beta$ is a function of the material and is generally larger than 2). In both models the presence and size of critical defects play a key role. However, defects cannot explain the colossal ductility (plastic deformation) seen in our experiments, nor can the presence of defects justify a size effect in an elastic property, namely Young's modulus. Only scarce evidence exists in the literature for similar (milder) size effects in epoxy fibers but without any structural justification. We find here that highly cross-linked necked epoxy fibers exhibit partial macromolecular anisotropy which likely explains the observed high mechanical characteristics.
\end{abstract}

Keywords: thermosetting resins, epoxy, mechanical properties, size effect, necking

\section{Introduction}

When measuring the physical properties of a material, a question of fundamental and practical significance arises: does the specimen size affect the value of the property measured? The answer to that question is important in practice since tests to establish values of, for example, material strength, are habitually performed with laboratory scale (thus comparatively small) specimens: if the strength decreases significantly with increasing specimen size, disastrous failures could occur if proper account were not taken of such size effect. Materials with reduced size and dimensionality such as thin fibers and films, nanotubes and nanowires, or metallic clusters indeed often exhibit exceptionally high mechanical properties compared to those of corresponding macroscopic specimens. In the field of solid mechanics it has long been known that the strength of materials depends on the volume of stressed material and on the nature of the stress distribution. Both of these effects usually arise because most materials are defect-sensitive, and defect severity and distribution are generally stochastic in nature: simply stated, the probability of finding a severe defect decreases with decreasing material volume, therefore large bodies tend to fail at lower stress levels than smaller ones when both are subjected to the same stress field, pure tension for example. The observation of such 'size effects' stretches 
back to Leonardo da Vinci who, in the 1500 s, described tests on different lengths of iron wires to measure how tensile strength reduces with increasing length [1]. A century later, Galileo understood and ingeniously described how structures behave differently on different scales [2]. After considering mechanical structures, he later extended the same logic to living organisms: he rationalized that because of the strength-size (or strength-weight) relationship, small animals are relatively stronger than large ones. In 1921 Griffith [3] perceived that the weakness of soda lime silica glass fibers was due to the presence of flaws and that a reduction in fiber diameter led to a significant increase in strength. In the second half of the $20^{\text {th }}$ century a number of studies were published on the issue of size effects, see [4-7] and references therein. Significant scale dependence is also observed for stiffness [8,9], and sometimes for toughness [5] of fibers (including nanofibers): this has important implications as to the potential role of micro- and nanomaterials as fibrous reinforcement in composites. The effect of size was measured by Cuenot et al. [8] for the elastic properties of silver and lead nanowires and of polypyrrole nanotubes. They attributed the increase of the apparent elastic modulus of polymer nanofibers to surface tension effects. Greenfeld and coworkers $[9,10]$ attributed the increase in stiffness, strength and toughness of nanofibers to the molecular orientation induced by the strong stretching of extensional flow. Only very few studies deal with size effects in epoxy resins, in spite of the relevance of the potentially higher mechanical properties of thin interfiber layers of matrix in a fiber reinforced composite, or of thin epoxy adhesives, or of epoxy coatings on $\mu \mathrm{m}$-sized fibers [10]. da Silva et al. [11] found that the fracture toughness of Araldite 2015 in Mode II increases with the adhesive thickness. Odom and Adams [12] tested dog-bone shaped specimens and observed a drastic increase in the strength of smaller specimens which they attributed to the reduction of flaw size. Hobbiebrunken et al. [13] proposed a method to produce $\mu \mathrm{m}$-sized epoxy fibers, the average strength of which was found to be remarkably close $(60 \%)$ to the theoretical strength. Leopold and coworkers $[14,15]$ used a similar experimental routine to investigate the size effect of carbonaceous fillers (graphene, carbon nanotubes and carbon black). In both studies the origin of the size effect on strength was attributed to the statistical distribution of defects. These authors also reported, without further discussion, the observation of a highly ductile behavior of their epoxy fibers. Recently Misumi et al. [16] studied the tensile behavior of fibers made of five different types of epoxies and compared the mechanical properties (yield stress, failure stress, failure strain, modulus and toughness) of fiber and bulk materials. Here too the epoxy fibers showed ductile behavior with a distinct yield point in all resin systems, whereas the macroscopic specimens exhibited brittle behavior with no yield point. Some epoxy micro-fiber specimens exhibited necking during the tensile test. The mechanical properties of the fibers were significantly higher (apart from the modulus) than those of their bulk counterparts but no diameter effect was observed (except for the failure strain) within the 100-150 $\mu \mathrm{m}$ diameter range of the fibers. Misumi et al. [16] recommended using microscale fiber test specimens, claiming that they provide a more realistic stressstrain response for describing the role of the matrix in composites at smaller length scales. Towse et al. [17] analyzed the defects causing failure and found a correlation between the size of the defects and the failure strain of an aerospace grade epoxy. Turk et al. [18] studied the potential of improved ductility of brittle epoxies for use in structural composites. Fiedler et al [19] observed relatively large plasticity in brittle epoxy (Toho-Rayon Ltd) resin specimens under shear. Thus, evidence on the size effect in epoxy fibers is scarce, and physical explanation is absent or just descriptive. It is therefore our aim to meticulously explore the size effects on stiffness, strength and failure strain for a wide range of fiber sizes, and to suggest possible mechanisms that may account for such cross-properties effects. In the present study we prepared a large number (more than 100) of diglycidyl ether of bisphenol-A (DGEBA) epoxy fibers with diameters ranging between 20 and $350 \mu \mathrm{m}$. The effects of diameter variation on the tensile strength, strain, modulus and fracture energy were examined and interpreted. Moreover, the observed high ductility and systematic occurrence of necking of the fibers, and the occurrence of a further size effect in fully necked fiber segments, were also studied and a prospective molecular level interpretation suggested.

\section{Experimental}

\subsection{Materials and preparation of specimens}

Diglycidyl ether of bisphenol-A (DGEBA), EP828 and hardener EP304 were purchased from Polymer$\mathrm{G}$, Israel. The curing agent/hardener EP304 is a 
polyether amine with trifunctional primary amine. Its amine groups are located on secondary carbon atoms at the ends of the aliphatic polyether chains. The resin and hardener were mixed in a glass vial in the weight ratio of 100:42, as recommended by the manufacturer. Both parts were manually stirred vigorously for $10 \mathrm{~min}$ to ensure homogeneous mixing, degassed for $30 \mathrm{~min}$, and then heated at $80^{\circ} \mathrm{C}$ in an oven for 24 minutes to almost reach the vitrified state, at viscosity optimized for the drawing of fibers. Subsequently, the mixture was taken out of the oven, a small amount of it was extracted from the reservoir using the $3 \times 5 \mathrm{~mm}$ tip of a spatula, and hung at a height of $\sim 1.5 \mathrm{~m}$ until a gradually thinner fiber formed under the effect of gravity, while gradually cooling down to ambient room temperature. The final diameter of the fibers was determined semi-empirically by modifying the amount and viscosity of the polymer collected at the tip of the spatula, such that lower amount and lower viscosity yielded thinner fibers and vice versa. The diameter at both ends was slightly larger than in the central region of the fiber, where it was uniform. The fibers were left to cure at room temperature for $48 \mathrm{hrs}$, then fixed on a rectangular metal frame $(8 \times 8 \mathrm{~cm})$ and post-cured for $5 \mathrm{hrs}$ at $80^{\circ} \mathrm{C}$ in an oven. The cured epoxy fibers had a smooth surface and of high quality (thus, free of surface defects), clear, transparent, and circular. They were subsequently cut into smaller specimens for testing. As already mentioned, the fibers to be tested had diameters between 20 to $350 \mu \mathrm{m}$ (most of them actually ranging from 40 to $160 \mu \mathrm{m}$ ), thus much wider than the $22-51 \mu \mathrm{m}$ range used by Hobbiebrunken et al. (2007) [13]. Moreover, dogboneshaped bulk epoxy samples were prepared in silicon molds using the same stoichiometric ratio, cured at $100^{\circ} \mathrm{C}$ for $6 \mathrm{hrs}$. It is noted that the curing processes of the bulk and fibers differed: the bulk was cured at the standard conditions recommended by the epoxy manufacturer, whereas the fibers were cured at lower temperatures over a longer duration in order to avoid overheating damage. Although the conditions were not identical, the comparison of the strength and stiffness of both samples is conservative as the higher temperature in the bulk samples would have been expected to yield higher cross-linking density and, consequently, strength and stiffness. As to the presence of defects, both samples were degassed to remove air microbubbles in order to avoid adverse effect on the mechanical properties.

\subsection{Differential scanning calorimetry (DSC)}

The thermal behavior of the epoxy samples was monitored by differential scanning calorimetry (DSC, TA DSC Q200). Samples were placed in a hermetically sealed aluminum pan. Measurements were carried in $\mathrm{N}_{2}$ atmosphere from room temperature to $300^{\circ} \mathrm{C}$, at a heating rate of $10^{\circ} \mathrm{C} \cdot \mathrm{min}^{-1}$. The glass transition temperature $\left(T_{\mathrm{g}}\right)$ is $83^{\circ} \mathrm{C}$ for the bulk epoxy and $80^{\circ} \mathrm{C}$ for the fibers. The amount of heat evolved from the (exothermic) curing process [20,21] was evaluated from the area of the exothermic peak. With the total heat associated with the curing of an uncured mixture considered as a reference, the residual heat was used to determine the percent cure of an incompletely cured sample, as shown by Equation (1):

Cure $[\%]=\frac{\Delta H_{\text {total }}-\Delta H_{\text {residual }}}{\Delta H_{\text {total }}} \cdot 100$

where $\Delta H$ is the area under the exothermal peak of the DSC curve.

\subsection{Mechanical testing of bulk specimens}

Bulk dogbone-shaped epoxy specimens, 1.50× $1.00 \mathrm{~mm}$ in cross-section and $15 \mathrm{~mm}$ in gauge length, were tested with a home-made mechanical testing system, using a $5 \mathrm{kN}$ load cell (Kistler 9311B, Switzerland) at $1 \mathrm{~mm} / \mathrm{min}$ crosshead speed. A total of 16 such macroscopic samples were tested. The nominal (or engineering) strain, $\varepsilon$, was determined from the crosshead displacement, and the nominal (engineering) stress was calculated by dividing the measured load $P$ by the initial cross-section.

\subsection{Mechanical testing of fiber specimens}

Two types of fibers were tested, as-prepared and fully necked fibers. The specimens of both types were cut and glued on windowed paper frames (gauge length: $10 \mathrm{~mm}$ ). Prior to testing, the diameter of each fiber was determined under an optical microscope by focusing on 3 points along the fiber, and the diameter value was averaged. Over 100 fibers of each type were prepared and measured. Mechanical tests were conducted with an Instron 5965 universal testing system (UK) equipped with a $10 \mathrm{~N}$ load cell, at a rate of $1 \mathrm{~mm} / \mathrm{min}$. The paper frame was held on the Instron with a pair of fiber clamps. Prior to testing the side edges of the frame (left and right) were cut out. As-prepared fibers were tested in tension and necking nucleated (usually at one of the clamps) at approximately $5 \%$ strain, eventually expanding 
through the entire gauge length, as will be discussed in the next section. Necked fibers were prepared by first stretching as-prepared fibers to a strain of $75 \%$, thus prior to the region of potential strain-hardening and/or failure, then cutting the resulting necked samples to $10 \mathrm{~mm}$ gauge length and mounting on paper frames as previously described. Following this, fully necked fiber segments were tested in tension under the same experimental conditions as the as-prepared fibers. In the remainder of the paper, 'as-prepared' fibers will simply be termed 'fibers' provided no ambiguity arises.

\section{Results and discussion}

Table 1 presents the average values of the bulk epoxy mechanical properties in tension and Figure 1 presents typical stress-strain curves of all epoxy specimen types. As seen, the mechanical signatures of bulk (macroscopic) and fibers (microscopic) epoxy specimens are radically unalike. In bulk specimens, a local instability sets in at the yield strength (or extension), closely followed by a drop in stress. At that point a neck begins to form (Figure 2a), leading to almost immediate failure at a nominal strength of $\sim 68 \mathrm{MPa}$ and a strain of $\sim 12 \%$. In sharp contrast, the as-prepared fibers begin to yield at a strain just under 5\%. Immediately after the yield point the stress somewhat

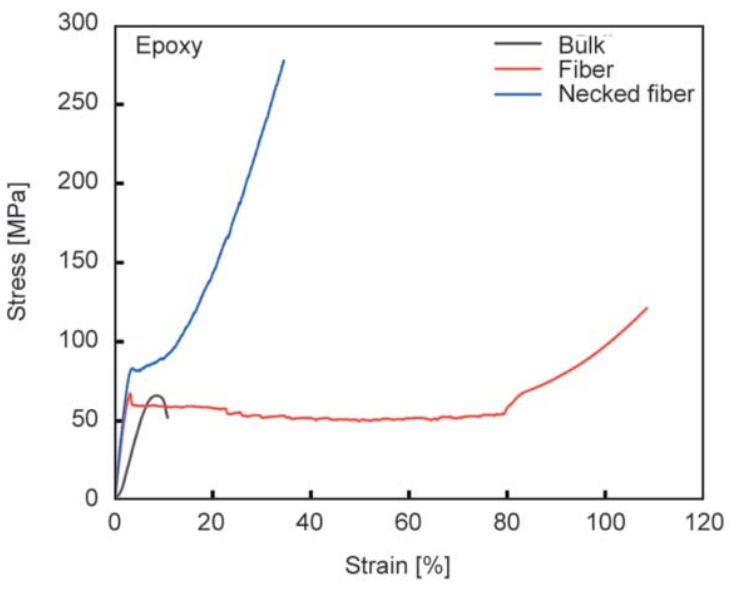

Figure 1. Typical stress-strain curves of bulk epoxy, as-prepared epoxy fiber, and necked epoxy fiber.

decreases with increasing deformation ('intrinsic strain softening'), followed by a plateau reflecting a lengthy necking phenomenon which, in most cases, propagates along the entire fiber length (Figure $2 b$ ). As a result, the average failure strain is $\sim 116 \%$ (sometimes even reaching $\sim 180 \%$ ). In later stages of the test the slope of the stress-strain curve increases ('strain hardening') and eventually the fiber fails at a nominal strength of $\sim 106 \pm 34 \mathrm{MPa}$ (the large variability is due to different fiber diameters). Such ductile behavior is more evocative of typical semi-crystalline polymers than of epoxy. Indeed, epoxy is an

Table 1. Average tensile mechanical properties of bulk epoxy samples.

\begin{tabular}{|c|c|c|c|c|c|}
\hline Epoxy specimens & $\begin{array}{c}\text { Modulus } \\
{[\mathbf{M P a}]}\end{array}$ & $\begin{array}{c}\text { Nominal strength } \\
{[\mathbf{M P a}]}\end{array}$ & $\begin{array}{c}\text { True strength } \\
{[\mathbf{M P a}]}\end{array}$ & $\begin{array}{c}\text { Failure strain } \\
{[\%]}\end{array}$ & $\begin{array}{c}\text { Toughness } \\
{[\mathbf{M P a}]}\end{array}$ \\
\hline Bulk & $1132.3 \pm 161.6$ & $67.8 \pm 2.0$ & - & $12.1 \pm 1.9$ & $5.1 \pm 1.1$ \\
\hline
\end{tabular}

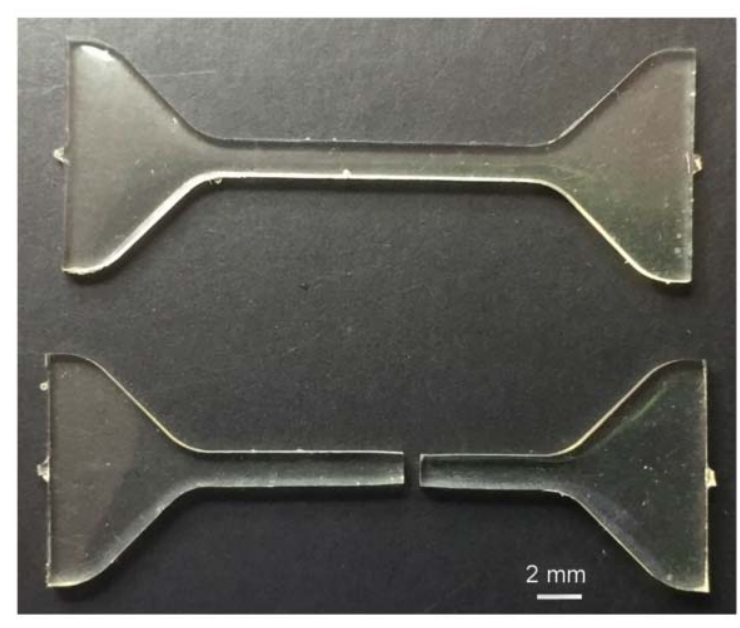

a)
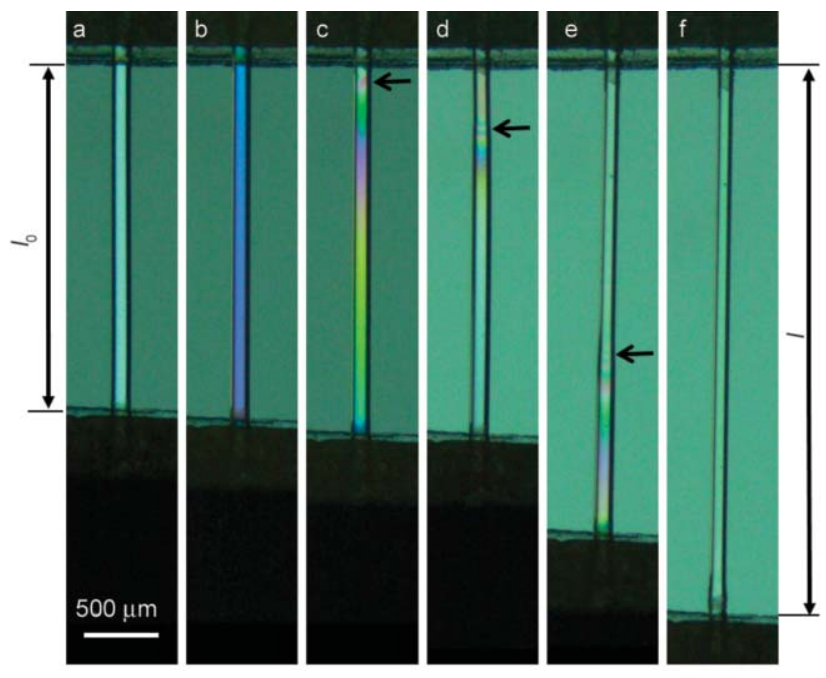

Figure 2. (a) Bulk epoxy specimen before and after a tensile test, note a beginning of necking at the fractured sides; (b) typical tensile test of as-prepared epoxy fiber (arrows designate the nucleation and propagation of necking), displaying the large elongation of the specimen (from $l_{0}$ to $l$ ). 
archetypal brittle amorphous isotropic polymer, which means that when stressed under a tensile force, its deformation is linear and elastic up to failure as shown in Figure 1 for a standard bulk sample (black line). The size effects on Young's modulus, strength, failure strain and toughness (the amount of energy per unit volume stored or absorbed up to fracture, quantified simply as the area under the stress-strain
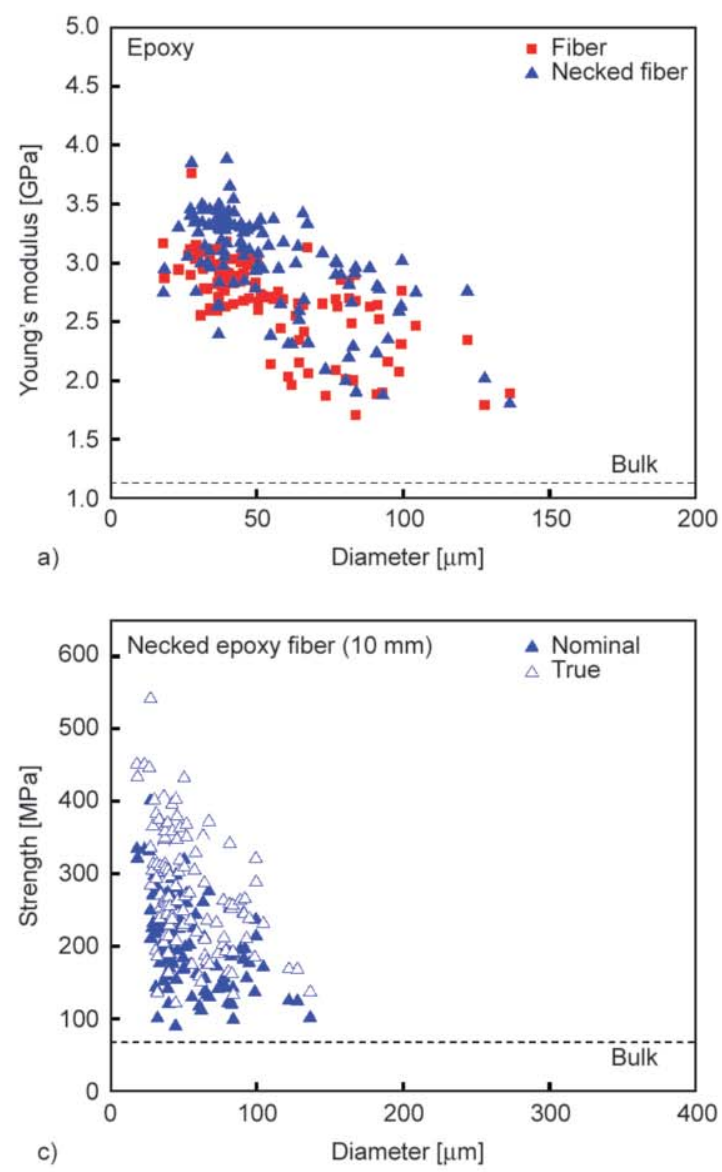

curve, thus $\int \sigma \mathrm{d} \varepsilon$, in $\mathrm{J} / \mathrm{m}^{3}=\mathrm{Pa}$ ), from bulk to fiber to necked fiber, are clearly significant (Figure 3 ).

DSC measurements (Figure 4) of the cured as-prepared epoxy fibers and bulk samples show that the fibers were $97 \%$ cured, compared to $100 \%$ curing in the bulk. Furthermore, the glass transition temperature dropped from $83^{\circ} \mathrm{C}$ in the bulk epoxy to $80^{\circ} \mathrm{C}$ in the as-prepared fibers. These observations may indicate
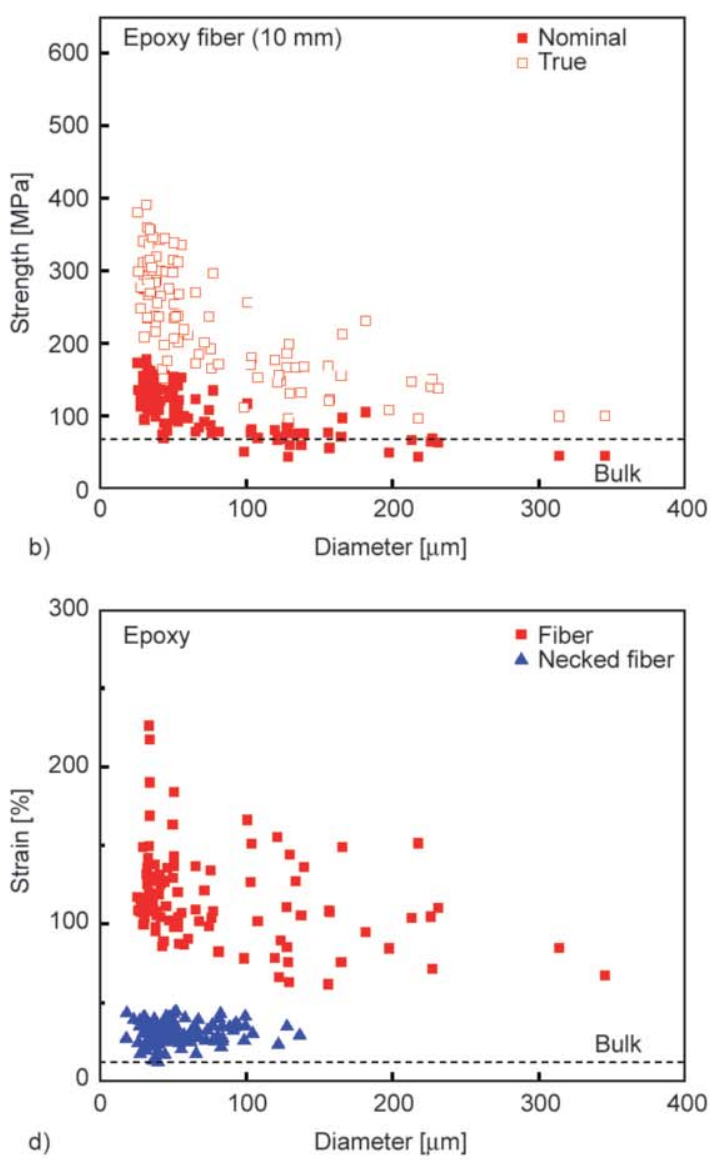

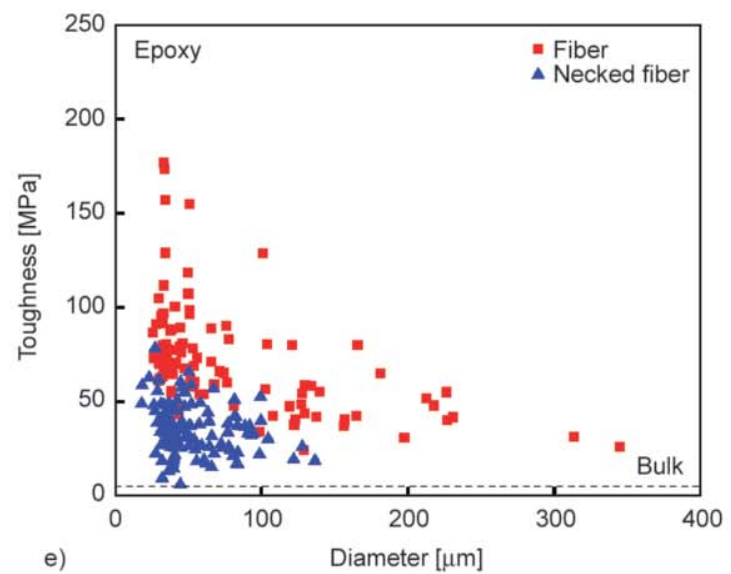

Figure 3. Size effects in epoxy (the horizontal dashed lines designate average bulk values): (a) dependence of Young's modulus on diameter of as-prepared and necked fibers; (b) dependence of (nominal and true) strength on diameter of as-prepared fibers; (c) dependence of (nominal and true) strength on diameter of necked fibers; (d) dependence of fracture strain on diameter of as-prepared and necked fibers; (e) dependence of toughness on diameter of as-prepared and necked fibers. 


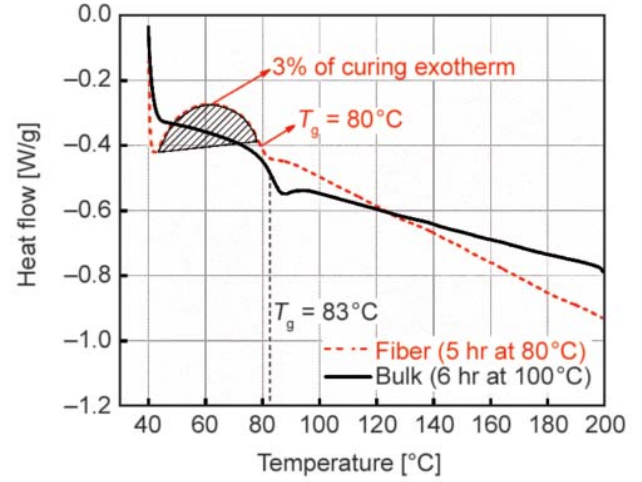

Figure 4. DSC curves of as-prepared epoxy fibers and bulk. The exothermic peak in the fibers sample indicates $97 \%$ curing, whereas no peak is observed in the bulk, indicating $100 \%$ curing. The glass transition temperature $T_{\mathrm{g}}$ is $80^{\circ} \mathrm{C}$ for the fibers and $83^{\circ} \mathrm{C}$ for the bulk.

some structural changes with respect to the bulk, such as higher chain mobility and therefore ductility of the as-prepared fibers (partially reflected in Figure $3 \mathrm{~d}$ ), but they seem too small to lead to the large increases in the observed mechanical properties. The crosslink density can in principle be evaluated from dynamic mechanical analyses but DMA testing is rather challenging with fiber specimens and requires extensive work, which we plan to accomplish in the near future. We performed wide-angle X-ray scattering (WAXS) measurements, described further on, which reveal molecular reorientation effects that are much more likely to lead to significant mechanical changes such as those observed here. As noted in the experimental section, the higher curing degree in the bulk samples compared to the fiber samples places our strength and stiffness improvement results on the conservative side. Further comprehensive DSC, WAXS and other experiments are anticipated in future research, to better understand the nature of the structural changes in the fibers.

In highly ductile specimens such as the as-prepared epoxy fibers studied here, it is more meaningful to use true stress values (thus, force normalized by the actual or instantaneous cross-section) than nominal (or engineering) stress (force normalized by the initial cross-section). Because of the cross-section contraction, true strength is always much higher than nominal strength, as seen in Figures $3 \mathrm{~b}$ and $3 \mathrm{c}$.

At constant gauge length $(10 \mathrm{~mm})$, the modulus and strength (and to a lesser extent, the strain and toughness) increase enormously with decreasing diameter $d$, as seen on Figure $3 \mathrm{~b}$ and $3 \mathrm{c}$. In particular, the strength closely approaches the conventional estimate of theoretical values, namely $\sim E / 10$ (see for example [22] on the link between the theoretical strength and modulus based on fracture mechanics), as seen by comparison with Figure 3a. Such diameter effect on strength is well known for many types of materials in fiber form, including polymers [9, 23-26]. The significant increase in the mechanical properties occurs below a certain critical diameter, $D_{\mathrm{c}}$, about $300 \mu \mathrm{m}$ for the as-prepared fiber and $150 \mu \mathrm{m}$ for the necked fiber (Figure 3), observed clearly in the strength and toughness plots. Such critical diameter is likely indicative of a structural transition, possibly above a certain mechanical drawing ratio, reminiscent of the polymer-network stretch transition in electrospun fibers [9]. Structural transition in mechanically drawn epoxy fibers could possibly be the consequence of overstretching and tearing of crosslinks, bundling of polymer segments, and/or internal confinement. The critical diameter $D_{\mathrm{c}}$ should entail material, process and structural parameters such as epoxy (resin and hardener) type, fraction and molecular structure, degree of curing, molecular microstructure, and internal friction, as well as surface quality and the size and density of defects. As the epoxy was the same for both fiber types, the observed difference in $D_{\mathrm{c}}$ is likely the result of a change induced in the molecular microstructure during the necking event. For example, the reduction in $D_{\mathrm{c}}$ caused by necking could be the result of tearing of crosslinks and thus lower effective internal friction between chain segments or bundles.

Results for the strength behavior of epoxy as a function of the fiber diameter can be elucidated in principle by means of a number of models $[6,23,26]$ either empirical (strength $\sim 1 / d$ ), or based on a classical fracture mechanics argument (strength $1 / d^{1 / 2}$ ), or via a Weibull model argument (strength $1 / d^{1 / \beta}$, where $\beta$, the Weibull modulus, is a function of the material and is generally larger than 2). In these models the presence and size of critical defects plays a key role. A comparison between the exponents of $d$ given by the LEFM and Weibull models is presented on a $\log$ (strength) vs $\log (d)$ experimental plot for the as-prepared epoxy fibers (Figure 5a) and for the necked fibers (Figure 5b). The latter were prepared by pre-straining epoxy fibers to about $80 \%$ of their original length (necking of the necked fibers was not observed). The necked fiber indeed exhibits higher mechanical properties in terms of strength and modulus. The nominal strength of the necked fiber 

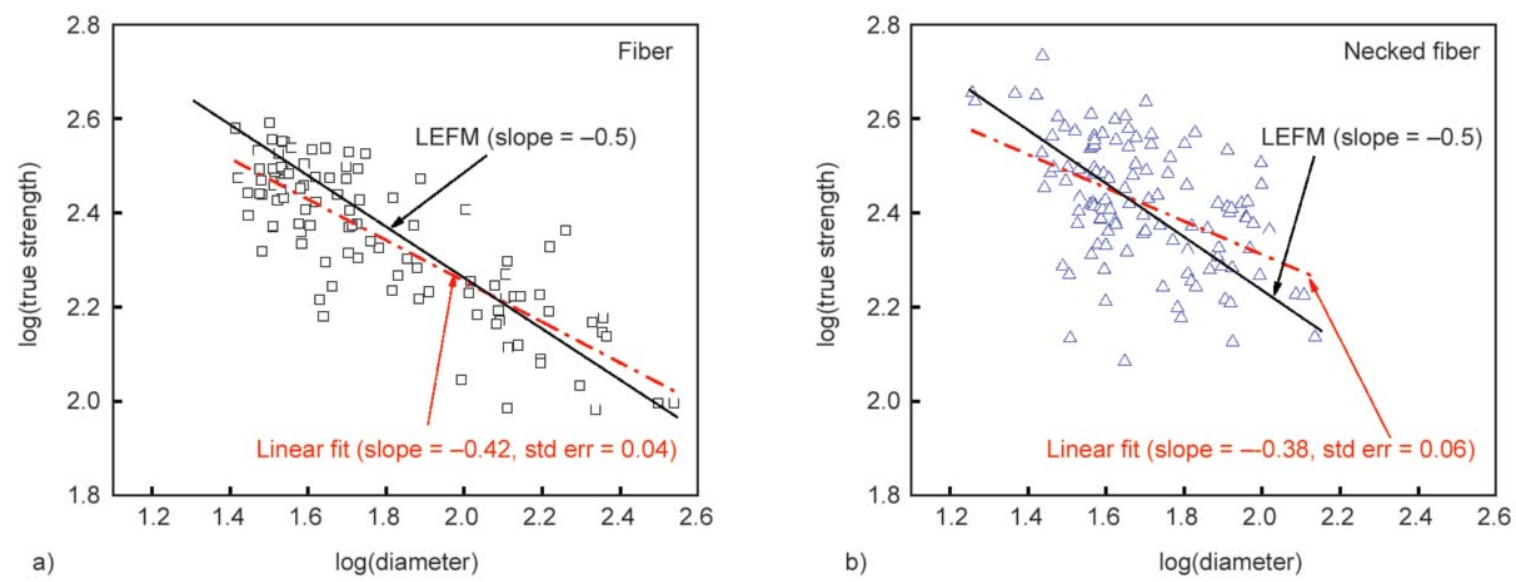

Figure 5. Log-log plot of strength against diameter for (a) as-prepared epoxy fibers and (b) necked fibers. In both cases the fracture mechanics prediction (slope $=1 / 2$ ) fits reasonably well the regression line through the data. Note the weaker fit of the line to the necked fiber data.

(204 MPa) is almost twice of that of as prepared fiber $(106 \mathrm{MPa})$, but the true strength only improves less than $20 \%$ (from 233 to $275 \mathrm{MPa}$ ).

The strength data in Figure 5 appear to fit the LEFM model reasonably well as for both fiber types the slope of the regression line through the data is close to 0.5 . The use of LEFM in this analysis is justified because, although the stretched fiber initially exhibits plastic behavior, towards its ultimate strength it becomes brittle due to strain hardening. This is evidenced by the fiber (red) stress-strain curve in Figure 1 , where after yield and up to $\sim 80 \%$ elongation the stress remains on a plateau, while at higher elongation up to fracture (that is, after necking is complete) the stress-strain slope rises to quasi linear elastic. Furthermore, the fracture surface (not shown) is distinctively smooth, typical of brittle fracture. As seen in Figure 5, the slope of the as-prepared fibers

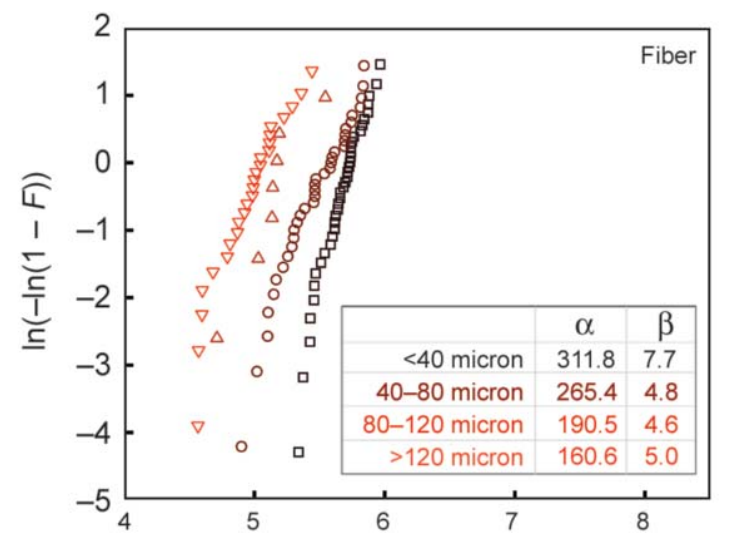

a)

In(true strength) is $-0.42 \pm 0.04$ and that of the necked fibers is $-0.38 \pm 0.06$. The higher dispersion of the strength data in the necked fibers compared to the as-prepared fibers is because all the necked fibers were prepared at the same drawing ratio of 1.75 as described in the methods section, regardless of the maximum achievable elongation of each fiber (thin fibers can elongate more than thick fibers). Note that the two slopes $(-0.42$ and -0.38$)$ give Weibull shape parameter values of 2.4 and 2.6 for the as-prepared fiber and the necked fiber, respectively. These values are about one-half or less than the shape parameter values obtained from direct Weibull plots as presented in Figure 6 and Table 2 where the data have been sorted out by diameter ranges. Those plots show parallel lines reflecting the difference in average strength for different diameters. The diameter effect on the scale parameter $\alpha$ (proportional to the average strength of the

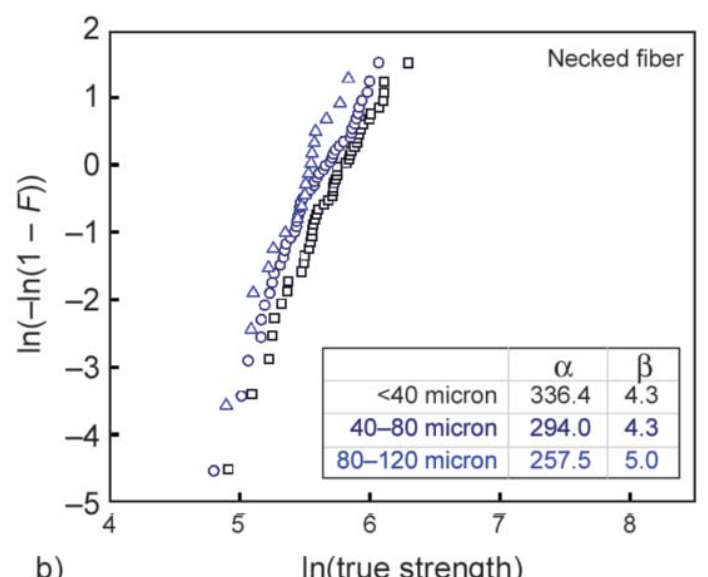

b)

Figure 6. Two-parameter Weibull distribution $F=1-\exp \left[-(\sigma / \alpha)^{\beta}\right]$ for (a) as-prepared epoxy fibers and (b) necked fibers. The data was separated into four diameter ranges: $<40$ micron, 40-80 micron, 80-120 micron and $>120$ micron. The effect of diameter on strength appears as a shift of the data; the values of the scale $(\alpha)$ and shape $(\beta)$ parameters are indicated in the inset, see also Table 2. 
Table 2. Summary of tensile data of epoxy fibers sorted by diameter ranges.

\begin{tabular}{|c|c|c|c|c|c|c|}
\hline & \multirow{2}{*}{$\begin{array}{c}\text { Diameter range } \\
{[\mu \mathrm{m}]}\end{array}$} & \multirow[b]{2}{*}{ No. of specimens } & \multirow{2}{*}{$\begin{array}{c}\text { Nominal } \\
\text { strength } \\
\text { [MPa] }\end{array}$} & \multirow{2}{*}{$\begin{array}{c}\text { True strength } \\
\text { [MPa] }\end{array}$} & \multicolumn{2}{|c|}{ Weibull distribution on true strength } \\
\hline & & & & & $\begin{array}{c}\alpha \\
{[\mathrm{MPa}]}\end{array}$ & $\beta$ \\
\hline \multirow{4}{*}{ As-prepared fibers } & $<40$ & 36 & $134.1 \pm 20.6$ & $295.0 \pm 45.3$ & 311.8 & 7.7 \\
\hline & $40-80$ & 33 & $112.0 \pm 26.3$ & $246.4 \pm 57.9$ & 265.4 & 4.8 \\
\hline & $80-120$ & 7 & $79.0 \pm 19.6$ & $173.8 \pm 43.1$ & 190.5 & 4.6 \\
\hline & $>120$ & 25 & $67.0 \pm 16.2$ & $147.3 \pm 35.6$ & 160.6 & 5.0 \\
\hline \multirow{4}{*}{ Necked fibers } & $<40$ & 46 & $227.1 \pm 63.4$ & $306.5 \pm 85.5$ & 336.4 & 4.3 \\
\hline & $40-80$ & 47 & $198.3 \pm 55.9$ & $267.6 \pm 75.5$ & 294.0 & 4.3 \\
\hline & $80-120$ & 18 & $175.1 \pm 40.3$ & $236.4 \pm 54.4$ & 257.5 & 5.0 \\
\hline & $>120^{*}$ & 3 & $117.0 \pm 13.5$ & $158.0 \pm 18.2$ & - & - \\
\hline
\end{tabular}

${ }^{*}$ Only 3 necked fiber specimens with diameter above 120 micron were available, therefore the Weibull statistical analysis was not performed.

population) is as expected in both cases, namely, the smaller the diameter the larger the scale parameter; however, the diameter effect on the shape parameter $\beta$ is not as clear in those plots, except for the obvious fact that the values of $\beta$ are at least double those in Figure 5. We conjecture here that direct Weibull plots (Figure 6) provide adequate values of the shape parameter but that the diameter effect on strength cannot be interpreted in a straightforward way in terms of the Weibull shape parameter as calculated from the $\log$ (strength) vs $\log$ (size) plot as in Figure 5. The reason for the discrepancy between the two types of plots is not obvious but is often observed [6, 24-26], including for other fiber types such as carbon. More sophisticated stochastic approaches discuss this and other aspects of size effects [6] but fall beyond the scope of the present paper.

From Table 1 and Figures $3 b$ and 3c, compared to the bulk value of epoxy strength ( $68 \mathrm{MPa})$, the microscale as-prepared epoxy fiber has higher nominal and true strengths, $106 \mathrm{MPa}$ and $233 \mathrm{MPa}$ on average, respectively. The strength increase in epoxy fiber is likely attributed to the following: (i) the surface of the fiber is almost defect-free since the formation of the fiber does not involve machining (ii) the small volume of the fiber limits the presence of critical defects in its interior. The additional strength observed in the necked fiber (nominal: $204 \mathrm{MPa}$, true: $276 \mathrm{MPa}$ ) is more difficult to explain since the necked fiber diameter is only slightly smaller than the as-prepared fiber diameter. An attempt to explain the additional strength increase is presented below, based on possible structural changes.

However, the presence of defects in a material cannot provide an explanation of a size effect in an elastic property, namely Young's modulus (Figure 3a), which for epoxy fiber is about 2.5 (as-prepared fiber) to 3 times (necked fiber) that of bulk epoxy. Nor can defects explain the colossal ductility (plastic deformation and failure strain) seen in our experiments with epoxy fibers. Only scarce evidence exists in the literature for similar (and milder) size effects in epoxy fibers $[13,16]$ but largely without explanation. We attempt to partly deal with this issue below.

Tensile toughness (or 'effective fracture energy', the amount of energy per unit volume that a material can store or absorb before fracture) was quantified simply as the area under the stress-strain curve as explained earlier. Compared to bulk epoxy, both epoxy fibers have higher strength and elongation, resulting in an order of magnitude increase in tensile toughness compared to bulk epoxy (Figure 3e).

Referring again to Figure 3a, the average value of Young's modulus of as-prepared epoxy fibers $(2.5 \mathrm{GPa})$ is more than twice that of bulk epoxy (1.1 GPa). Moreover, following necking, it is further improved to $3.0 \mathrm{GPa}$. As previously noted, diameter effects on Young's modulus have previously been observed in semi-crystalline polymers and other fiber types at the micron-size scale $[6,23-26]$ as well as in various sub-micron sized electrospun polymer fibers [9, 27-30]. The necking phenomenon seen here in epoxy is almost never-observed in highly cross-linked glassy polymers. It is accompanied by an increase in Young's modulus with decreasing diameter which cannot be explained by the presence of defects (which for the fiber strength leads to a $d^{-1 / 2}$ dependence as discussed earlier). It is postulated here that the ductility, necking behavior, and size effect in highly cross-linked epoxy fibers might be linked, if not attributed, to macromolecular re-alignment during necking. This is a highly counterintuitive 
conjecture because, unlike semi-crystalline polymers, or even lightly cross-linked polymers, epoxy is an amorphous polymer which forms 3D rigid interconnects when cured, thus a macromolecular network with very little propensity to flow [31] and reorient. In Figure 7, however, we present wide-angle X-ray scattering (WAXS) evidence of molecular (re)orientation in necked epoxy fibers, whereas epoxy films, and as-prepared and tensile tested fibers show no preferred orientation. Herman's orientation parameter, calculated from the WAXS data for the necked fibers, is $\left.f=1.5<\cos ^{2} \alpha\right\rangle-0.5=0.23 \pm 0.04$, where $\alpha$ is the angle between the fiber axis and the macromolecules. Since $f$ can vary between 0 (no preferred orientation) and 1 (full alignment with the fiber axis), this result clearly indicates molecular orientation. Although the orientation data exhibit a weak increasing trend with respect to decreasing fiber diameter, as might be expected, this trend should be validated by further experiments.

As the as-prepared fibers do exhibit diameter dependence in both their stiffness and toughness, it could be
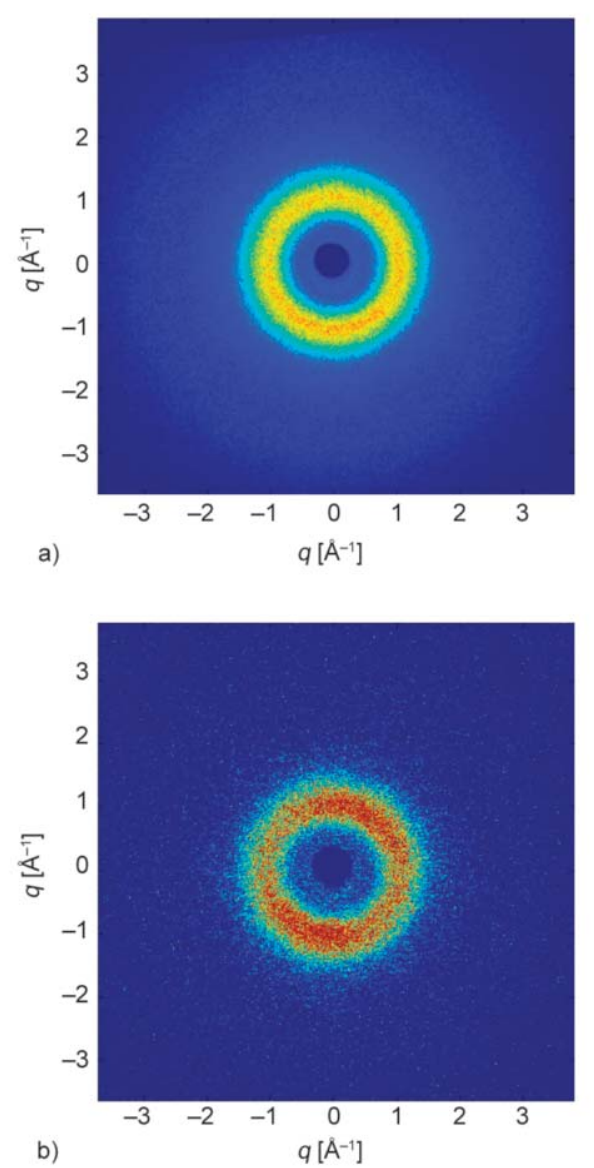

that their degree of alignment was below the WAXS detection threshold. Efforts are currently underway to expand these preliminary WAXS observations. If molecular (re)orientation in necked epoxy fibers is indeed confirmed, an explanation/mechanism will have to be proposed for this surprising result. This would likely involve reordering of polymer segments, distortion and/or loss of crosslinks (evidenced in the DSC measurements of the as-prepared fibers), or other molecular-scale structural changes, which would favor mechanisms leading to high strength, stiffness and plasticity.

In that context, regarding necked fibers, the notion of size dependence may be somewhat misleading, as it implies a direct influence of the fiber diameter on the tensile mechanical properties. Rather, the diameter and the strength/modulus/toughness are separately dependent on the mechanical drawing ratio during fiber preparation; thus, the fundamental property is the drawing ratio instead of the diameter. In other words, a high drawing ratio reduces the fiber diameter while at the same time increases the
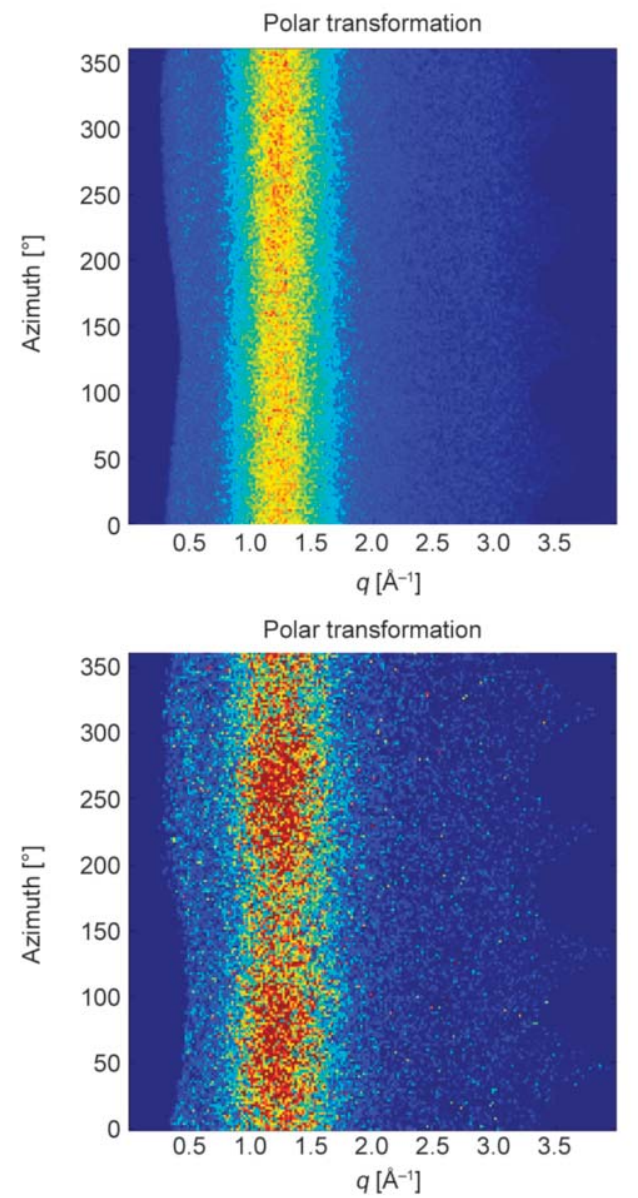

Figure 7. Wide-angle X-ray scattering patterns of (a) an as-prepared epoxy fiber, showing no detected evidence of preferred orientation at the sub-nanometer scale, and (b) a necked epoxy fiber where structural orientation (arc-shapes) is clearly visible. $q$ is the scattering vector $\left[\AA^{-1}\right]$, and color reflects intensity (arbitrary units). 
molecular orientation and consequently the strength and elastic modulus.

\section{Conclusions}

Epoxy fibers with different diameters were prepared and their stiffness, strength, ultimate strain, and tensile toughness demonstrated substantial scale-dependent effects as they all significantly increased with a decrease in diameter. Surprisingly, below a critical diameter the mechanical behavior changed from defect-controlled to a yield and plastic deformationcontrolled strengthening, showing an extreme ductile behavior compared to bulk epoxy. Necked fiber segments subsequently tested in tension were found to have even higher strength and modulus compared to the initial as-prepared fibers. Size effects for the strength of epoxy can be elucidated in principle either by means of a classical fracture mechanics argument or via a stochastic model argument. In both of these approaches the presence and size of critical defects plays a key role. However, defects cannot explain the extensive plastic deformation seen in our experiments, or justify a size effect in Young's modulus, an elastic property. Only scarce evidence exists in the literature for similar (milder) size effects in epoxy fibers but without any structural justification. We present here first indications (by WAXS) that highly cross-linked necked epoxy fibers exhibit macromolecular anisotropy which likely explains the observed high mechanical characteristics.

\section{Acknowledgements}

The electron microscopy studies were conducted at the Irving and Cherna Moskowitz Center for Nano and Bio-Nano Imaging at the Weizmann Institute of Science. The authors gratefully acknowledge Dr. ing. Gerhard Kalinka from Bundesanstalt für Materialforschung und -prüfung (BAM), Berlin, Germany, for his skilled assistance with the mechanical testing device.Thanks are due to Dr. Ellen Wachtel and Dr. Yishay Feldman (Chemical Research Support, Weizmann Institute of Science) for their expert assistance with preliminary X-ray tests. This research was made possible in part by the generosity of the Harold Perlman family. H.D.W. is the recipient of the Livio Norzi Professorial Chair in Materials Science. This work is supported by the German Research Foundation (DFG) in the project 'Evaluation and modelling of the fatigue damage behavior of polymer composites at reversed cyclic loading' (project \#281870175).

\section{References}

[1] da Vinci L.: Codex Atlanticus. folio 222 (1486-1490).

[2] Galilei G.: Dialogues concerning two new sciences (1638). Dover Publications, New York (1954).

[3] Griffith A. A.: The phenomena of rupture and flow in solids. Philosophical Transactions of the Royal Society of London, Series A, 221A, 163-198 (1921). https://doi.org/10.1098/rsta.1921.0006

[4] Irwin G. R.: Fracture. in 'Elasticity and plasticity' (ed.: Flügge S.) Springer-Verlag, Berlin, Vol 6, 551-588 (1958).

[5] Carpinteri A.: Scaling laws and renormalization groups for strength and toughness of disordered materials. International Journal of Solids and Structures, 31, 291302 (2004).

https://doi.org/10.1016/0020-7683(94)90107-4

[6] Wagner H. D.: Stochastic concepts in the study of size effects in the mechanical strength of highly oriented polymeric materials. Journal of Polymer Science Part B: Polymer Physics, 27, 115-149 (1989).

https://doi.org/10.1002/polb.1989.090270108

[7] Bažant Z. P.: Size effect on structural strength: A review. Archive of Applied Mechanics, 69, 703-725 (1999).

https://doi.org/10.1007/s004190050252

[8] Cuenot S., Fretigny C., Demoustier-Champagne S., Nysten B.: Surface tension effect on the mechanical properties of nanomaterials measured by atomic force microscopy. Physical Review B, 69, 165410/11654101/5 (2004).

https://doi.org/10.1103/PhysRevB.69.165410

[9] Greenfeld I., Sui X., Wagner H. D.: Stiffness, strength, and toughness of electrospun nanofibers: Effect of flow-induced molecular orientation. Macromolecules, 49, 6518-6530 (2016).

https://doi.org/10.1021/acs.macromol.6b01204

[10] Greenfeld I., Zhang W. Y., Sui X., Wagner H. D.: Intermittent beading in fiber composites. Composites Science and Technology, 160, 21-31 (2018). https://doi.org/10.1016/j.compscitech.2018.03.003

[11] da Silva L. F. M., de Magalhães F. A. C. R. G., Chaves F. J. P., de Moura M. F. S. F.: Mode II fracture toughness of a brittle and a ductile adhesive as a function of the adhesive thickness. The Journal of Adhesion, 86, 891905 (2010).

https://doi.org/10.1080/00218464.2010.506155

[12] Odom E. M., Adams D. F.: Specimen size effect during tensile testing of an unreinforced polymer. Journal of Materials Science, 27, 1767-1771 (1992). https://doi.org/10.1007/BF01107202

[13] Hobbiebrunken T., Fiedler B., Hojo M., Tanaka M.: Experimental determination of the true epoxy resin strength using micro-scaled specimens. Composites Part A: Applied Science and Manufacturing, 38, 814818 (2007). https://doi.org/10.1016/j.compositesa.2006.08.006 
[14] Leopold C., Liebig W. V., Wittich H., Fiedler B.: Size effect of graphene nanoparticle modified epoxy matrix. Composites Science and Technology, 134, 217-225 (2016). https://doi.org/10.1016/j.compscitech.2016.08.022

[15] Leopold C., Augustin T., Schwebler T., Lehmann J., Liebig W. V., Fiedler B.: Influence of carbon nanoparticle modification on the mechanical and electrical properties of epoxy in small volumes. Journal of Colloid and Interface Science, 506, 620-632 (2017).

https://doi.org/10.1016/j.jcis.2017.07.085

[16] Misumi J., Ganesh R., Sockalingam S., Gillespie J. W.: Experimental characterization of tensile properties of epoxy resin by using micro-fiber specimens. Journal of Reinforced Plastics and Composites, 35, 1792-1801 (2016). https://doi.org/10.1177/0731684416669248

[17] Towse A., Potter K., Wisnom M. R., Adams R. D.: Specimen size effects in the tensile failure strain of an epoxy adhesive. Journal of Materials Science, 33, 4307 4314 (1998).

https://doi.org/10.1023/A:1004487505391

[18] Turk M., Hamerton I., Ivanov D. S.: Ductility potential of brittle epoxies: Thermomechanical behaviour of plastically-deformed fully-cured composite resins. Polymer, 120, 43-51 (2017). https://doi.org/10.1016/j.polymer.2017.05.052

[19] Fiedler B., Hojo M., Ochiai S., Schulte K., Ando M.: Failure behavior of an epoxy matrix under different kinds of static loading. Composites Science and Technology, 61, 1615-1624 (2001). https://doi.org/10.1016/S0266-3538(01)00057-4

[20] Barton J. M.: The application of differential scanning calorimetry (DSC) to the study of epoxy resin curing reactions. in 'Epoxy resins and composites I. Advances in polymer science'. Springer, Berlin, Vol 72, 111-154 (1985). https://doi.org/10.1007/3-540-15546-5 5

[21] Ma S., Liu W., Zhao Y., Yan Z., Gao N.: Curing behavior and thermal properties of autocatalytic cycloaliphatic epoxy. Journal of Macromolecular Science Part A: Pure and Applied Chemistry, 49, 81-84 (2012). https://doi.org/10.1080/10601325.2012.630971
[22] Yuan C. C., Xia X. K.: On the correlation of Young's modulus and the fracture strength of metallic glasses. Journal of Applied Physics, 109, 033515/1-033515/5 (2011). https://doi.org/10.1063/1.3544202

[23] Galiotis C., Young R. J.: The solid-state polymerization and physical properties of bis(ethyl urethane) of 2,4hexadiyne-1,6-diol: 3. Mechanical properties. Polymer, 24, 1023-1030 (1983). https://doi.org/10.1016/0032-3861(83)90155-6

[24] Smook J., Hamersma W., Pennings A. J.: The fracture process of ultra-high strength polyethylene fibres. Journal of Materials Science, 19, 1359-1373 (1984).

https://doi.org/10.1007/BF01120049

[25] Wagner H. D., Steenbakkers L. W.: Stochastic strength and size effect in ultra-high strength polyethylene fibres. Philosophical Magazine Letters, 59, 77-85 (1989). https://doi.org/10.1080/09500838908214780

[26] Wagner H. D.: Dependence of fracture stress upon diameter in strong polymeric fibers. Journal of Macromolecular Science Part B: Physics, 28, 339-347 (1989). https://doi.org/10.1080/00222348908215228

[27] Ji Y., Li B. Q., Ge S. R., Sokolov J. C., Rafailovich M. H.: Structure and nanomechanical characterization of electrospun PS/clay nanocomposite fibers. Langmuir, 22, 1321-1328 (2006). https://doi.org/10.1021/la0525022

[28] Sui X., Greenfeld I., Cohen H., Zhang X., Li Q., Wagner H. D.: Multilevel composite using carbon nanotube fibers (CNTF). Composites Science and Technology, 137, 35-43 (2016). https://doi.org/10.1016/j.compscitech.2016.10.011

[29] Sui X., Wagner H. D.: Tough nanocomposites: The role of carbon nanotube type. Nano Letters, 9, 1423-1426 (2009). https://doi.org/10.1021/n1803241y

[30] Sui X., Wiesel E., Wagner H. D.: Enhanced mechanical properties of electrospun nano-fibers through $\mathrm{NaCl}$ mediation. Journal of Nanoscience and Nanotechnology, 11, 7931-7936 (2011). https://doi.org/10.1166/jnn.2011.4760

[31] van Krevelen D. W., te Nijenhuis K.: Properties of polymers: Their correlation with chemical structure; Their numerical estimation and prediction from additive group contributions. Elsevier, Amsterdam (2009). 